\title{
Differential Evolution in Wireless Communications: A Review
}

\author{
https://doi.org/10.3991/ijoe.v15i11.10651 \\ Hilary I. Okagbue ${ }^{(\triangle)}$ \\ Covenant University, Ota, Nigeria \\ hilary.okagbue@covenantuniversity.edu.ng \\ Muminu O. Adamu \\ University of Lagos, Lagos, Nigeria \\ Timothy A. Anake \\ Covenant University, Ota, Nigeria
}

\begin{abstract}
Differential Evolution (DE) is an evolutionary computational method inspired by the biological processes of evolution and mutation. DE has been applied in numerous scientific fields. The paper presents a literature review of DE and its application in wireless communication. The detailed history, characteristics, strengths, variants and weaknesses of DE were presented. Seven broad areas were identified as different domains of application of DE in wireless communications. It was observed that coverage area maximisation and energy consumption minimisation are the two major areas where DE is applied. Others areas are quality of service, updating mechanism where candidate positions learn from a large diversified search region, security and related field applications. Problems in wireless communications are often modelled as multiobjective optimisation which can easily be tackled by the use of DE or hybrid of DE with other algorithms. Different research areas can be explored and DE will continue to be utilized in this context.
\end{abstract}

Keywords-Differential evolution, multiobjective optimisation, evolutionary computation, energy utilisation, localisation, coverage, wireless networks.

\section{Evolutionary Computation}

Physical phenomena are routinely studied using models. The models are sometimes complex because of different parameters that constitute them. At times, the analysis of such models is prohibitively complex and waste computation time. The insights and benefit accrual from the analysis of complex models have pushed researchers to look for a viable alternative method, which is nature [1]. Natural processes have inspired researchers in the development of computational processes, methods and algorithm that can be used to solve complex methods or to provide the best available (optimum) solution of their models. [2]. Because of the complexity of some models, optimisation became the only alternative since the models have different candidate solutions [3]. 
Optimisation is central to many natural processes as the method of evolution, memes and adaptation implies that organisms attempt to adjust over time to fit in optimally to their environment despite the constraints of space, search for food, shelter, and search for mates and so on. The study of these natural processes gave birth to the notion of computational intelligence of which evolutionary computation is a subfield [4].

EC is a family of iterative algorithms inspired mostly by biological evolution and are employed mostly in global optimisation [5]. In addition, is a branch of applied mathematics that deals with the global optimisation of a given function or set of functions based on some predefined criteria. Global optimisation focuses on finding the maximum or minimum of all input values while local optimisation deals with finding local minima or maxima.

In EC, an initial set of candidate solutions is generated and iteratively updated to minimise or maximise the given function [6]. Each new solution (generation) is produced by stochastically removing weaker or less desired solutions - survival of the fittest and iteratively introducing random changes until a termination criterion that guarantee a feasible solution is obtained [7]. EC techniques can produce highly optimized solutions given a wide range of constraints and complex objective function [8]. This makes EC a suitable tool for solving multi-dimensional problems and advanced optimisation [9].

The choice of EC is mainly based on the nature of the problem to be solved and the corresponding data structures. EC techniques perform well in solving higher procedure problems that are designed to find, select or search or determine a heuristic (partial search algorithm) that obtain a near optimal solution [10]. EC works with incomplete or partial, or imperfect information and limited completion capacity [11]. However, EC does not guarantee that an optimal (exact) solution will be obtained [12].

Differential evolution (DE) is one of the most widely used EC technique [13-15]. The biological processes of evolution, mutation and adaptation inspired the development of DE.

The aim of this review is to critically analyse the different areas where DE has been applied in wireless communications.

\section{Differential Evolution}

The basic procedure of Differential evolution (DE) is given. A population of candidate solutions (called vectors) is moved around in the search space by using the mathematical formulae defined for the objective (fitness) function to combine the positions of existing vectors from the population. If the new position of the vector is an improvement, then it is accepted and promoted to form part of the population. Otherwise, the new position is simply discarded and sometimes archived. The process is repeated t times until a satisfactory solution is discovered. Note that the algorithm does not guarantee that an optimal (exact) solution will be found.

Let $f: R^{n} \rightarrow R$, be the fitness function, which must be minimised, based on some equality, inequality or bounded constraints. The function maps a candidate solution in the form of vector in a given dimensional space to a real number as output, 
which indicates, the fitness of the given candidate solution. The aim is to find $\mathrm{m}$ for which $f(m) \leq f(p)$ for all $\mathrm{p}$ in the search space of the given dimension, which means that the solution $\mathrm{m}$ is the only global minimum available.

The detailed development, component, variants, application areas and application of DE in curve fitting are presented here.

\subsection{Historical development}

The DE algorithm was created to be a very vital force in evolutionary computing. The first articles on DE was basically introductory [16] and it took a year later to actually demonstrate the computational efficiency of the method in an IEEE international contest built in conference [17], where it tops other EA in solving real-valued functions. Researchers were interested in the method and some papers were published to further elucidate the theory, processes and mathematics behind the method, which give birth to a clearer DE algorithm. The papers are [16] and [18-19]. Since then, DE has proven to be a force to be reckoned with, in EC. Summary of the method can be found in [20] and [21], and their references therein.

In the application of DE, the individual candidate (trial) solutions are called parameter vectors or genomes. DE utilizes difference of the parameter vectors to assess and explore the objective function landscape, which is a deviation from the other EA. The reliance of DE on difference vectors can be traced to earlier algorithms which uses difference vectors in their computation. The algorithms are Nelder-Mead algorithm [22] and the controlled random search algorithm [23].

According to $[24[$, the reasons why DE is used by researchers as a reliable optimisation tools are listed.

1. DE is simpler and easier to implement when compared with other evolutionary algorithms. The code is easy to code with different programming languages and can easily be understood by novices.

2. The performance is better than most EC in terms of convergence, computational speed, accuracy and robustness [25]. This makes it a suitable candidate for handling unimodal, multimodal, homogenous, non-homogenous, separable and nonseparable systems [26].

3. The number of control parameters (Cr, F, and NP) in DE is very few. This has helped to reduce the computational burden associated with the method [27].

4. The low space complexity of DE has been helpful in the use of DE for solving large scale, nonlinear and multi-dimensional optimisation problems.

\subsection{Control parameters of the differential evolution}

There are three main control parameters of the DE algorithm: the mutation scale factor F, the crossover constant $\mathrm{Cr}$ and the population size NP [24]. A good choice of the control parameters is necessary for the efficient execution of the DE [28]. It is recommended that for the one-dimensional case, the least population size is 4 , mutation factor is set as 0.5 and the crossover constant should be adjusted between 0 and 1 
and this has to be problem specific since some objective functions tend to be sensitive to the choice of the control parameters [29]. Wrong choice of these parameters will ultimately frustrate the algorithm, leading to slow convergence speed and inaccurate results $[30]$.

\subsection{Different variants of differential evolution}

Since the introduction of DE, researchers have continued to propose different variants of the algorithm without necessarily altering its foundation. The nonlinear and complex nature of some problems have led researchers to push back the boundaries of DE. The different variants of DE are listed.

Differential evolution using trigonometric mutation:This was proposed by [31], aimed at speeding up the performance of DE by splitting the target vectors [32].

Differential evolution using arithmetic recombination: This comes as a departure from the traditional binomial crossover used in DE. Here, recombination can be either continuous or arithmetic. The trial vector is now expressed as a linear combination of the components from the donor and target vectors [33]. Furthermore, the coefficients of the combination can be a random variable or constant [34].

DE/rand/1/either-or algorithm: This variant of the DE algorithm was designed in such a way that the here the trial vectors that are pure mutants and those that are pure recombinants are mutually exclusive [34]. This method appears to perform better than the classical DE [25].

Opposition based differential evolution (ODE): DE usually start with some random guesses and works with no prior information about the actual optimum solution [35]. Fast convergence to the optima can be obtained simultaneously by checking the fitness of the opposite solution [36]. This has proved useful as the initial candidate solution can be chosen between the better fit options of the guess or opposite guess [37]. The process can be extended before the birth of each individual of the population.

Differential evolution with neighborhood-based mutation: This is the use of exploitation in DE which helps the algorithm to search new regions in a multidimensional search space. This variant of DE is effective because of two things. Firstly, the search algorithm utilizes the initial information and search towards the optima and lastly, the search algorithm will be very effective in the introduction and management of information into the population [38]. The idea behind this to prevent the search from favouring only vectors in the neighbourhood, but to extend the search to other areas.

Differential evolution with adaptive selection of mutation strategies: This variant of DE makes use of the control parameters values and self-adapted trial vector $g$ eneration strategies to produce new individuals that are candidates for the optimum solution [27]. This is done by using the historical data and creating patterns that will generate the solution (unsupervised learning).

Adaptive DE with DE/current-to-pbest mutation: This uses a specific external data learning tool (archive) or algorithm to relate and interact effectively with the records of failure and success and subsequently update the control parameters with the 
information. This helps in the creation of new candidate solutions to facilitate convergence and to discourage arbitrary tuning of the control parameters [26].

Hybrid Differential Evolution Algorithms: Hybrid models or algorithms in general are the combination of two or more parent algorithms to produce another one (offspring), whereby the outcome (offspring) is expected to be better than the parent algorithms. This is because; hybrid algorithms are built upon the best features of the parent algorithms. Hybridisation in DE takes three forms.

- Hybridisation with other EC algorithms: such as particle swarm optimisation [3940], cultural algorithms [41], biogeography-based optimisation [42], earthworm [43], bacterial foraging optimisation algorithm [44-45], bare bones [46] and modified bare bones swarm optimizers [47], ant bee colony algorithm [48] and genetic algorithm [49]. Others are: tissue membrane systems [50], artificial immune systems [51], firefly algorithm [52], simulated annealing [53], neural networks [54], bat algorithm [55], krill herd algorithm [56], memetic inspired systems [57], fireworks optimisation [58], Cuckoo Search algorithm [59] and Grey Wolf optimizer [60].

- The use of local search technique (algorithms) in DE to improve the ability of the $\mathrm{DE}$ algorithm to make effective utilization of the information collected and to push towards obtaining the optimum solution [61]. The local search is usually adapted in the crossover stage of the DE, thereby increasing the likelihood that the optimal solutions (offspring with high fitness) will be found in small neighbourhood around the candidate solutions and reduction of fitness function evaluations. Hybridisation can also be done using neighbourhood search [62], Taguchi operator [63], clustering [64], shuffled [65] and chaotic local search, Levy flight, and Golden Section Search [66].

- Hybridisation with non-Darwinian methods: DE has been hybridized with some non-evolutionary techniques such as: black hole inspired systems [67] and gravitation search algorithm [68] and radial basis function response surface [69].

- Differential Evolution for Discrete and Binary Optimisation: DE was originally created for real value parameters. Over the years, several researchers have modified it to be utilized in tackling binary and discrete optimisation problems. This can be achieved by the following: truncating or approximating the parameter values for objective function evaluation [70] or discretisation of continuous value parameters [71], bicriteria [72] and purely binary optimisation [73]. Most applications of DE in this context are for solving job and machine scheduling problems.

Parallel differential evolution: DE can be modified to solve problems concurrently, which can be in hardware or software modes. This is done by breaking complex problems into small bits [74]. Speed, accuracy and reducing the complexity of objective function evaluation is the motivation behind parallel DE [75]. The other EC algorithm can be employed for more accuracy [76]. Parallel DE ensures that the heterogenous nature of the optimal population is preserved after migrations. In addition, the method allows for solving optimisation problems with mixed integer and real parameter values [77]. 


\subsection{Major strengths of the differential evolution}

DE can be applied to tackle real world problems, which are unimodal/multimodal, linear/non-linear, differentiable/nondifferentiable convex/non-convex, continuous/ non-continuous and symmetrical/asymmetrical in nature. These are categorized as follows:

Multiobjective optimisation (MO): Real world problems are often complex because of the composition of the different variables that are used in modelling them. These imply some problems have several criteria and objectives, which must be evaluated simultaneously in order to obtain the solution [78]. DE has proven to be well suited for tackling this type of optimisation problem. The most prominent modified version of DE that is used in solving MO problems are Pareto DE [79] and non-Pareto DE [80].

Constrained optimisation (CO): DE is very good at solving real world problems that come with conditions known as constraints. The most profound constraints are called the boundary constraint [81] or boundary value problems in numerical analysis or numerical optimisation and inequality constraints [82].

Large-scale optimisation (LSO): Most search abilities of EC algorithms failed at a very high dimension. This is due to, firstly, the complexity of the problem and the fatigue of the search strategy and secondly, the exponential increase in the solution space caused by the time it takes for the search to yield an optimum solution. This problem is present in almost all the EC algorithm of which DE is not an exception. However, experts in DE have provided means of which DE can be used to solve large scale optimisations. Some of the surveyed approaches are the fitness function refinement [83], use of chaotic systems and simplex search method [84], co-evolution [85], self-adaptive method [86], random grouping scheme [87], surrogate-assisted [88] and hybrid of co-evolution and log-normal self-adaptation [89], fuzzy adaptive method [90], strategy adaptation [27] and competition based strategy [91].

Optimisation in dynamic and uncertain environments: EC algorithms generally suffers from the uncertainties present in the optimisation problems. Those uncertainties can be time, place or measurement indexed. These uncertainties can manifest as the noisiness of the fitness function, the effect of the computing environment on the parameters, case of the fitness function being approximated and the optimal nature of the candidate solution varies over time and location. Researchers have designed different strategies to address these issues in DE environment. Intermittent varying of the scale parameter and incorporated a very good search method [92], optimisation of the objective functions that are slow and also changes with time [93] and introduction of aging mechanism to handle unstable fitness functions [94] are some of the available strategies.

Multimodal optimisation and niching: Most of objective functions encountered in real life are multimodal in nature and as such caution must be exercised in handling them because their nature connotes that several near optimal solutions may be available for them. Researchers have proposed different niching methods to tackle this issue. Niching ensures that multiple groups are maintained within the same population in order to track different optimum solutions. Some niching techniques include, but 
not limited to the following: fitness sharing [95], clearing [96], crowding [97], speciation [98] and restricted tournament selection [99].

\subsection{Challenges and future research areas of differential evolution}

Irrespective of the advancement made in the modification of DE, the method is still faced with some challenges, some of which are presented.

- $\mathrm{DE}$ is still struggling to tackle objective functions that are not linearly separable [100].

- DE has been observed to fail to adequately convey the population to large distances across the solution spaces, especially when clustered population of candidate solutions are encountered [101].

- Rotation invariance remains an issue [102].

- DE is often plagued with low convergence rate due to the action of the randomized mutation operators and competition between the population and its individuals [103].

- DE is yet to convincingly prove that it can compute expensive problems better than other evolutionary computation methods [104].

- It is still very vague in DE environment; the optimum population size adaptation strategy to adopt that will yield optimum performance [105].

- Learning based approaches (supervised, reinforcement and unsupervised learning) have not been fully incorporated in DE [106].

- The problem of parameter settings indicates that more research is needed in this direction [107].

- The search continues for an EC that can guarantee $100 \%$ optimum solution.

- The following are yet to be fully developed or estimated for DE. They include: computational complexity, convergence rate estimation, expected first hitting time, the necessary and sufficient conditions that guarantee convergence and unified formulation in the theoretical development [107].

- It cannot be stated, the ranking performance of DE in solving these problems which include: multiobjective optimisation, constrained optimisation, large-scale optimisation, optimisation in dynamic and uncertain environments or multimodal optimisation.

- Finally, it can be seen that there is no automatic method of selecting a DE variant for a given problem since studies have shown that DE variants are designed to improve one aspect of DE and as such, may perform very well for a specific type of problem and perform very poor in others. Fan et al. [108] has proposed an autoselection mechanism (ASM) in order to tackle the challenge.

\section{DE in Wireless Communications}

Wireless communication encompasses all processes and procedures that guarantee the transfer of information over a distance without physical connection between the 
two or more points, that is, without the aid of wires, cables or any other forms of electrical conductors. The transmitted distance ranges from a few metres to thousands of kilometres. Wireless communication can be used in radio wireless technology, cellular telephony, wireless internet access, satellite and broadcast television, wireless home networking, cordless telephones and others. Wireless communication has several advantages over wired one. It is cost effective, flexible, convenient, fast, easily accessible and the probability of maintaining constant connectivity is high. However, security, coverage and other challenges are some of the issues with wireless communication.

The problems encountered in wireless communication are often NP hard. Consequently, they are modelled as a constrained optimisation problem, of which differential evolution (DE) is routinely applied to optimize the objective function resulting from the problem formulation. Minimisation or maximisation of the objective function subject to some constraints are usually the main.

A review of previous studies showed that there are six interconnecting areas where DE has been applied in wireless communications. They are: energy optimisation, improving quality of service, localisation and coverage area maximisation, updating mechanism, security application and related field applications.

\subsection{Energy optimisation}

Energy is required in transmission of data in wireless networks and estimation of energy consumption is important for network planning [109]. Energy consumption optimisation is a predictor of overall network performance and remains the most important constraint. DE has been used to achieve efficient energy optimisation in WSN [110] and power allocation in orthogonal frequency division multiplexing (OFDM) systems [111] thereby decreasing the gross impact of the limited available energy [112].

In order to maintain consistent energy, energy harvesting technology has been proposed to improve network throughput. DE is used to obtain the optimal throughput that will sustain consistent energy [113] and extend the lifetime of individual nodes in WSNs [114].

Delay in forwarding packets of data is a strategy to ensure efficient energy consumption, which can be achieved by providing the optimum solution of clustering and routing in wireless sensor networks (WSN) using DE [115]. Clustering ensures that data is transmitted in hierarchical order and reduces into distinct groups which helps to improve power utilization. DE has been used in clusters optimisation and an effective energy optimisation strategy [116] which guarantees network longevity [117] and optimum packet delivery ratio [118]. A hybrid of DE and simulated annealing has been used as clustering algorithm and it achieves the set goals of efficient energy utilization by reduction of loss of cluster heads and sustaining network lifetime [119].

DE was applied in the power consumption minimisation in Long Term Evolution (LTE) base stations [120]. 


\subsection{Quality of service improvement}

In improving the quality of network, it is always desirable to optimize the constraints that will yield maximum quality of service (QOS). DE has been applied to the optimisation of network coverage, power consumption, cost and human exposure minimisation to the network [121-122]. Different examples are given. They are: the case of heterogenous networks consisting of WiFi access points [123]; multi objective node deployment to ensure reliable and efficient real time performance [124-125] and lifetime maximisation [126]; optimum allocation of spectrum in wireless networks [127] and minimisation of the number of links in WSNs [128].

DE has been applied to minimize the installation cost satisfying QOS constraints in Wireless Mesh Network (WMN) [129] and minimisation of overall mobility management cost in wireless cellular networks [130]. Others are the minimisation of design and transmission costs with the aid of DE [131].

The following QOS constraints were optimised using DE; the bit error rate (BER), bandwidth, associativity-based routing (ABR), monetary cost and signal to noise ratio (SNR) [132]. For instance, a hybrid of DE and genetic algorithm was used to minimise the BER and multi-path effect of the channel thereby increasing the convergence speed [133-134]. Also, DE was used to minimize the BER in Multi-User Multiple Input Multiple Output (MU-MIMO) [135] and in general, solving the beamforming problems subjected to different variables and constraints [136]. DE was used in the optimum allocation of bandwidth in Cellular IP network, thereby improving the QoS [137].

The consequence of the optimisation is the minimisation of hangovers, that is the "Ping Pong" effect [138], minimisation of end to end video reconstruction distortion [139] and resilience strategy which guarantees that a network can withstand the failure of few a nodes or links. Relay nodes is one of the resilience strategy and DE is used to find the optimum number of relay nodes that will improve connectivity and minimize network downtime [140].

Multicast routing is often preferred strategy in quality service delivery, especially in multichannel multiradio wireless mesh networks. DE has shown to be efficient in finding the optimal performance in routing [141-142], packet delivery ratio maximisation [143], delay minimisation [144] and optimum reassigning vacant channel to cognitive users without network deterioration [145]. Assignment can also take the form of allocation in download link systems [146].

Transmission rate, transmitter location and network throughput of different wireless networks have been optimized using the DE [147]. Application of DE helped in the optimisation of network throughput in networks with dynamic topological structure [148].

In order to improve network performance, DE was applied in the reporting cell problem (RCP), antenna positioning problem (APP) [149], antenna synthesis problem used in Near Field Communication (NFC) technologies [150-151] and optimisations of channel state information [152] and geometry of coupler [153]. 


\subsection{Localisation and coverage area maximisation}

Localisation in wireless networks often involves the location of sensed data in wireless sensors and devices. Location information on localisation is crucial in coverage, sensor node deployment, target tracking and routing. DE was applied as a localisation algorithm to enhance the quality of information and for convergence purposes of determining the optimal distances between nodes [154-155]. Location quality can be enhanced using DE [156-157] and specifically in base stations (BS) [158]. Apart from accuracy of location estimation, $\mathrm{DE}$ has shown to be useful in reducing the time complexity, thereby leading to localisation error reduction [159-160]. A further reduction of the localisation error was achieved by the hybrid of DE and Monte Carlo localisation algorithm. This is a case where the sample weight is taken as the objective function [161]. A hybrid of DE and genetic algorithm has been used as a localisation algorithm in the estimation of the location of nodes in WSN [162]. Moreover, localisation by using DE can be improved by adaptive controls over the parameters to ensure adequate tuning [163].

Generally, coverage problems in WSN are modelled as optimisation problem and can be solved using evolutionary algorithms such as DE [164]. DE is used in solving connection based localisation problem features prominently in wireless sensor networks where connections can be modelled as a nonconvex or non-convex optimisation problem which can easily be handled using DE [165].

DE has been used in finding the minimum subset of sensor nodes to cover all the targets in wireless multimedia sensor networks [166] and hence solving the targets coverage problem [167-168] and nudge redundant active nodes into sleep mode [169] or reduction of number of individual nodes which participate in non-dominated solution sorting [170]. Also available is the use of DE to optimize sensor nodes over diverse area shape, thereby increasing the coverage area [171]. Coverage radius and load balancing were optimized using DE which acts as the gateway deployment algorithm [172]. DE was used as deployment algorithm in optimisation of variables defined for directional WSNs [173].

\subsection{Updating mechanism}

DE algorithm can be applied as position updating mechanism where candidate positions learn from a large diversified search region such as online heuristic searching [174] and search equations for the purpose of reliable data collection [175]. The outcome is to determine the optimum path that satisfies the different quality of service (QOS) constraints in Mobile Ad Hoc Networks (MANETs) [176]. DE has been found to be a crossover strategy used as a search tool in solving optimisation problems in WSNs and superior to genetic algorithm and particle swarm optimisation [177].

DE was used to compute the fitness function in a hybrid algorithm aimed at finding the optimal path for efficient data transmission in WSN based air pollution monitoring system [178]. 


\subsection{Security}

Security issues are one of several issues facing WSN and intrusion detection system (IDS) is indispensable in the security of WSN. The aim of IDS is to detect malicious activities that affect the predefined network protocols. The multi-dimensional nature of the datasets of IDS causes data redundancy which leads to poor performance and slow speed. In order to address the data dimension issue, feature selections are often used in IDS which can be effectively optimised by the application of DE [179]. Apart from IDS, trust interference is another method of addressing security issues in WSN. DE was applied to compute trust values for each individual node in the WSN [180].

Another aspect of the security issues in WSN is the data aggregation caused by an enormous connectivity from different devices connected to the network. As a result, the network is vulnerable to security threats at the aggregated nodes. To solve the problem, DE was used to compute the trusted aggregated node among multiple nodes [181]. DE was combined with artificial immune system in the optimisation of the distribution and effectiveness of the detector generator in WSN intrusion detection [182].

The strategy of maintaining network reliability and at the same time achieving privacy preservation in WMAN can be handled using IoT-oriented offload method. DE can be used to optimize the variables while preserving privacy [183]. Random flipped is often recommended in preventing security attacks of WSN on an insecure link. Optimum flipping can be obtained using DE to minimize the fusion error and ensuring secure data transmission [184]. DE was applied to obtain optimal power schedule in wireless networks thereby, minimizing the occurrence of the denial of service (DoS) attacks [185].

\subsection{Related field applications}

DE is applied when the studied problem is modelled as a network with given objective function to be minimised or maximised. Another aspect is when DE is combined with other methods and applied in fields related to wireless communication. DE was applied to determine the optimal design for the appropriate pipes that fits the network distribution in water distribution system subject to cost and total loss constraints [186].

DE was applied to predict gas concentration while WSN systems were used to collect the data [187]. DE was used in energy optimisation of environmental driven WSN [188]. DE is used in path planning for unmanned underwater vehicle's (UUV) [189].

\section{Conclusion}

Differential evolution (DE) is one of the most widely applied evolutionary computational technique. The properties of DE outlined in this review, makes the algorithm, a favourable choice in the optimisation in wireless communications. Different variants 
of DE have been used in wireless communication. Two major strengths of DE are efficient handling of multi-objective and multimodal optimisations. As seen in the review, most of the optimisation problems in wireless networks are multi-objective and multimodal in nature, of which DE was applied to obtain the desired solutions. It appears that coverage area maximisation and energy consumption minimisation are the two major areas where Differential Evolution is applied in wireless networks and communications, which was part of the submission of [190]. The networks are usually modelled as a multi-objective optimisation problem where variables are optimised using some constraints. The variables can be energy efficiency, coverage, resource allocation and so on, while the constraints can be in the form of link conflict and interference [191]. Thereafter, evolutionary algorithms, DE in this case are applied to solve the optimisation problem subject to the given constraint. Different research paths on the use of DE in wireless networks can be followed.

\section{Acknowledgement}

The authors are grateful to Covenant University for providing an enabling environment for this research.

\section{References}

[1] Fogel, D.B. (1995). Evolutionary Computation: Toward a New Philosophy of Machine Intelligence. IEEE Press, Piscataway, NJ.

[2] Bäck, T., Fogel, D. B. \& Michalewicz, Z. (Eds), (1997). Handbook of Evolutionary Computation. Oxford University Press.

[3] De Jong, K.A. (2006). Evolutionary Computation: A Unified Approach. MIT Press, Cambridge MA.

[4] Eiben, A.E. \& Smith, J.E. (2003). Introduction to Evolutionary Computing. Springer.

[5] Fogel, L.J., Owens, A.J. \& Walsh, M.J. (1966). Artificial Intelligence through Simulated Evolution, New York: John Wiley.

[6] Rechenberg, I. (1971). Evolutionsstrategie - Optimierung Technischer Systeme nach Prinzipien der Biologischen Evolution. PhD thesis. https://doi.org/10.1002/fedr.491086050 $\underline{6}$

[7] Holland, J.H. (1975). Adaptation in Natural and Artificial Systems. University of Michigan Press, Ann Arbor.

[8] Koza, J.R. (1992). Genetic Programming: On the Programming of Computers by Means of Natural Evolution. MIT Press, Massachusetts.

[9] Diaz Ochoa, J.G. (2018). Elastic Multi-scale Mechanisms: Computation and Biological Evolution. J. Molec. Evol. 86 (1): 47-57.

[10] Jamshidi M. (2003). Tools for intelligent control: fuzzy controllers, neural networks and genetic algorithms. Philos. Trans. Royal Soc. A. 361 (1809): 1781-808.

[11] Danchin, A. (2008). Bacteria as computers making computers. FEMS Microbiol. Review, 33 (1): 3-26.

[12] Onwubolu, G. C., \& Babu, B. V. (2014). New optimization techniques in engineering. Springer-Verlag Berlin Heidelberg. ISBN: 978-3-540-20167-0. 
[13] Ighravwe, D.E., Oke, S.A. \& Adebiyi, K.A. (2018). An enhanced reliability-oriented workforce planning model for process industry using combined fuzzy goal programming and differential evolution approach. J. Ind. Engine. Int., 14(1): 185-212. https://doi.org/10. 1007/s40092-017-0223-9

[14] Ighravwe, D.E. \& Oke, S.A. (2016). A machine survival time-based maintenance workforce allocation model for production systems. Afr. J. Sci. Tech. Innovat. Develop., 8(5-6): 457-466.

[15] Adebiyi, A.A. \& Ayo, C.K. (2015). Portfolio selection problem using generalized differential evolution 3. Appl. Math. Sci., 9(41-44): 2069-2082.

[16] Storn, R. \& Price, K.V. (1995). Differential evolution: A simple and efficient adaptive scheme for global optimization over continuous spaces, ICSI, USA, Tech. Rep. TR-95012. [Online]. Available: http://icsi.berkeley.edu/ storn/litera.html.

[17] Storn, R. \& Price, K.V. (1996). Minimizing the real functions of the ICEC 1996 contest by differential evolution. In Proc. IEEE Congress Evol. Comput., 842-844. https://doi.org $\underline{\text { /10.1109/icec.1996.542711 }}$

[18] Price, K.V. (1997). Differential evolution vs. the functions of the 2nd ICEO. In Proc. IEEE Congress Evol. Comput., 153-157.

[19] Price, K.V. \& Storn, R. (1997). Differential evolution: A simple evolution strategy for fast optimization. Dr. Dobb's J., 22(4): 18-24.

[20] Eltaeib, T. \& Mahmood, A. (2018). Differential evolution: A survey and analysis. Appl. Sci., 8(10): Art. no. 1945.

[21] Javaid, N. (2019). Differential evolution: An updated survey. Adv. Intel. Syst. Comput., 772: 681-691.

[22] Nelder, J.A. \& Mead, R. (1965). A simplex method for function minimization. Computer J., 7: 308-313. https://doi.org/10.1093/comjnl/7.4.308

[23] Price, W.L. (1977). Global optimization by controlled random search, Computer J., 20(4): 367-370.

[24] Das, S. \& Suganthan, P.N. (2011). Differential evolution: A survey of the state-of-the-art. IEEE Trans. Evol. Comput., 15 (1): 4-31.

[25] Das, S., Abraham, A., Chakraborty, U.K. \& A. Konar, A. (2009). Differential evolution using a neighborhood-based mutation operator. IEEE Trans. Evol. Comput., 13(3): 526-553. https://doi.org/10.1109/tevc.2008.2009457

[26] Zhang, J. \& Sanderson, A.C. (2009). JADE: Adaptive differential evolution with optional external archive. IEEE Trans. Evol. Comput., 13 (5): 945-958. https://doi.org/10.1109 tevc.2009.2014613

[27] Qin, A.K., Huang, V.L. \& Suganthan, P.N. (2009). Differential evolution algorithm with strategy adaptation for global numerical optimization. IEEE Trans. Evol. Comput., 13(2): 398-417. https://doi.org/10.1109/tevc.2008.927706

[28] Liu, J. \& Lampinen, J. (2002). On setting the control parameters of the differential evolution method. In: Matoušek, R. \& Ošmera, P. (eds.) Proc. Mendel, 8th Int. Conf. Soft Comput., 11-18.

[29] Liu, J. \& Lampinen, J. (2002). Adaptive parameter control of differential evolution. Matoušek, R. \& Ošmera, P. (Eds.) Proc. Mendel, 8th Int. Conf. Soft Comput., 19-26.

[30] Gämperle, R., Müller, S.D. \& Koumoutsakos, P. (2002). A parameter study for differential evolution. In Proc. WSEAS Int. Conf. Adv. Intel. Syst. Fuzzy Syst. Evol. Comput., 293298.

[31] Fan, H.Y. \& Lampinen, J. (2003). A trigonometric mutation operation to differential evolution. J. Global Optimiz., 27(1): 105-129. 
[32] Vaishali, Sharma, T.K., Abraham, A. \& Rajpurohit, J. (2018). Enhanced asynchronous differential evolution using trigonometric mutation. Adv. Intel. Syst. Comput., 614: 386-397.

[33] Price, K.V. (1999). An introduction to differential evolution, In New Ideas in Optimization, D. Corne, M. Dorigo, \& V. Glover, Eds. London, U.K.: McGraw-Hill, 79-108.

[34] Price, K.V., Storn, R. \& Lampinen, J. (2005). Differential Evolution-A Practical Approach to Global Optimization. Berlin, Germany: Springer.

[35] Tizhoosh, H.R. (2005). Opposition-Based Learning: A New Scheme for Machine Intelligence, Int. Conf. on Comput. Intel. for Model. Control and Auto.- I, 695-701, Vienna, Austria.

[36] Rahnamayan, R.S., Tizhoosh, H.R. \& Salama, M.M.A. (2008). Opposition-based differential evolution. IEEE Trans. Evol. Comput., 12 (1): 64-79. https://doi.org/10.1109/te vc. 2007.894200

[37] Tizhoosh, H.R. (2006). Opposition-based reinforcement learning, J. Adv. Comput. Intel. Intel. Inform., 10: Article: 3.

[38] Mezura-Montes, E., Vel'azquez-Reyes, J. \& Coello, C.A. (2006). A comparative study of differential evolution variants for global optimization, In Proc. Conf. Genetic Evol. Comput., 485-492. https://doi.org/10.1145/1143997.1144086

[39] Xin, B., Chen, J., Peng, Z.H. \& Pan, F. (2010). An adaptive hybrid optimizer based on particle swarm and differential evolution for global optimization. Sci. China, Series F: Info. Sci., 53(5): 980-989. https://doi.org/10.1007/s11432-010-0114-9

[40] Xin, B., Chen, J., Zhang, J., Fang, H. \& Peng, Z.-H. (2012). Hybridizing differential evolution and particle swarm optimization to design powerful optimizers: A review and taxonomy. IEEE Trans. Syst. Man Cyber. Part C: Appl. Rev., 42 (5): 744-767. https://doi.org/10.1109/tsmcc.2011.2160941

[41] Awad, N.H., Ali, M.Z., Suganthan, P.N. \& Reynolds, R.G. (2017). CADE: A hybridization of Cultural Algorithm and Differential Evolution for numerical optimization. Info. Sci., 378: 215-241. https://doi.org/10.1016/j.ins.2016.10.039

[42] Gong, W., Cai, Z. \& Ling, C.X. (2011). DE/BBO: A hybrid differential evolution with biogeography-based optimization for global numerical optimization. Soft Comput.,15(4): 645-665. https://doi.org/10.1007/s00500-010-0591-1

[43] Javaid, N., Ullah, I., Zarin, S.S., Kamal, M., Omoniwa, B. \& Mateen, A. (2019). Differential-evolution-earthworm hybrid meta-heuristic optimization technique for home energy management system in smart grid. Adv. Intel. Syst. Comput., 773: 15-31. https: //doi.org/10.1007/978-3-319-93554-6 2

[44] Biswas, A., Dasgupta, S., Das, S. \& Abraham, A. (2007). A synergy of differential evolution and bacterial foraging optimization for global optimization. Neural Network World, 17(6): 607-626.

[45] Passino, K.M. (2002). Biomimicry of bacterial foraging for distributed optimization and control. IEEE Control Syst. Mag., 22(3): 52-67. https://doi.org/10.1109/mcs.2002.1004010

[46] Omran, M.G.H., Engelbrecht, A.P. \& Salman, A. (2009). Bare bones differential evolution. Euro. J. Operat. Res., 196(1): 128-139.

[47] Zhang, Q., Zhang, H., Yang, B. \& Hu, Y. (2018). Gaussian Cauchy differential evolution for global optimization. Comm. Comput. Info. Sci., 888: 166-182

[48] Jadon, S.S., Tiwari, R., Sharma, H. \& Bansal, J.C. (2017). Hybrid Artificial Bee Colony algorithm with Differential Evolution. Appl. Soft Comput. J., 58: 11-24. https://doi.org/10. 1016/j.asoc.2017.04.018

[49] Trivedi, A., Srinivasan, D., Biswas, S. \& Reindl, T. (2015). Hybridizing genetic algorithm with differential evolution for solving the unit commitment scheduling problem. Swarm Evol. Comput., 23: 50-64. https://doi.org/10.1016/j.swevo.2015.04.001 
[50] Zhang, G., Cheng, J., Gheorghe, M. \& Meng, Q. (2013). A hybrid approach based on differential evolution and tissue membrane systems for solving constrained manufacturing parameter optimization problems. Appl. Soft Comput. J., 13(3): 1528-1542. https://doi.org/ 10.1016/j.asoc.2012.05.032

[51] Lin, Q., Zhu, Q., Huang, P., Chen, J., Ming, Z. \& Yu, J. (2015). A novel hybrid multiobjective immune algorithm with adaptive differential evolution. Comp. Oper. Res., 62: 95-111. https://doi.org/10.1016/j.cor.2015.04.003

[52] Abdullah, A., Deris, S., Mohamad, M.S. \& Hashim, S.Z.M. (2012). A new hybrid firefly algorithm for complex and nonlinear problem. Adv. Intel. Soft Comput., 151: 673-680. https://doi.org/10.1007/978-3-642-28765-7 81

[53] Addawe, R.C., Addawe, J.M., Sueno, M.R.K. \& Magadia, J.C. (2017). Differential evolution-simulated annealing for multiple sequence alignment. J. Physics: Conf. Series, 893(1): 012061. https://doi.org/10.1088/1742-6596/893/1/012061

[54] Wang, L., Zeng, Y. \& Chen, T. (2015). Back propagation neural network with adaptive differential evolution algorithm for time series forecasting. Expert Syst. Appl., 42(2): 855863. https://doi.org/10.1016/j.eswa.2014.08.018

[55] Fister Jr., I., Fister, D. \& Yang, X.-S. (2013). A hybrid bat algorithm. Electrotechnical Review, 80(1-2): 1-7.

[56] Wang, G.-G., Gandomi, A.H., Alavi, A.H. \& Hao, G.-S. (2014). Hybrid krill herd algorithm with differential evolution for global numerical optimization. Neural Comput. Appl., 25(2): 297-308. https://doi.org/10.1007/s00521-013-1485-9

[57] Wu, X. \& Che, A. (2019). A memetic differential evolution algorithm for energy-efficient parallel machine scheduling. Omega, 82: 155-165. https://doi.org/10.1016/ j.omega.2018.01.001

[58] Zheng, Y.-J., Xu, X.-L., Ling, H.-F. \& Chen, S.-Y. (2015). A hybrid fireworks optimization method with differential evolution operators. Neurocomputing, 148: 75-82. https://doi.org/10.1016/j.neucom.2012.08.075

[59] Lin, H. \& Siu, S. (2018). A Hybrid Cuckoo Search and Differential Evolution Approach to Protein-Ligand Docking. Int. J. Molec. Sci., 19(10): Article 3181. https://doi.org/ 10.3390/ijms19103181

[60] Gupta, S. \& Deep, K. (2019). Hybrid Grey Wolf Optimizer with mutation operator. Adv. Intel. Syst. Comput., 817: 961-968.

[61] Noman, N. \& Iba, H. (2008). Accelerating differential evolution using an adaptive local search. IEEE Trans. Evol. Comput., 12(1): 107-125. https://doi.org/10.1109/tevc.2007.895272

[62] Yang, Z., Tang, K. \& Yao, X. (2007). Differential evolution for high dimensional function optimization. In Proc. IEEE Congress Evol. Comput., 3523-3530.

[63] Yildiz, A.R. (2013). Hybrid Taguchi-differential evolution algorithm for optimization of multi-pass turning operations. Appl. Soft Comput. J., 13(3): 1433-1439. https://doi.org/ $\underline{10.1016 / j . a s o c .2012 .01 .012}$

[64] Cai, Z., Gong, W., Ling, C.X. \& Zhang, H. (2011). A clustering-based differential evolution for global optimization. Appl. Soft Comput. J., 11(1): 1363-1379. https://doi.org/ $\underline{10.1016 / j . a s o c .2010 .04 .008}$

[65] Srinivasa Reddy, A. \& Vaisakh, K. (2013). Shuffled differential evolution for large scale economic dispatch. Elect. Power Syst. Res., 96: 237-245. https://doi.org/10.1016/ j.epsr.2012.11.010

[66] Mishra, I., Mishra, I. \& Prakash, J. (2019). Differential evolution with local search algorithms for data clustering: A comparative study. Adv. Intel. Syst. Comput., 742: 557-567. 
[67] Sharma, P., Sharma, H., Kumar, S. \& Sharma, K. (2019). Black-hole gbest differential evolution algorithm for solving robot path planning problem. Adv. Intel. Syst. Comput., 741: 1009-1022. https://doi.org/10.1007/978-981-13-0761-4_95

[68] Li, X., Yin, M. \& Ma, Z. (2011). Hybrid differential evolution and gravitation search algorithm for unconstrained optimization. Int. J. Phys. Sci., 6(25): 5961-5981.

[69] Deng, K. \& Chen, H. (2017). Hybrid Optimization Algorithm based on Differential Evolution and RBF Response Surface. Chinese J. Theo. Appl. Mech., 49 (2): 441-455.

[70] Lampinen, J. \& Zelinka, I. (1999). Mixed integer-discrete-continuous optimization with differential evolution. In Proc. of Mendel, 5th Int. Conf. on Soft Comput., 71-76.

[71] Tasgetiren, M.F., Liang, Y.C., Sevkli, M. \& Gencyilmaz, G. (2004). Differential Evolution Algorithm for Permutation Flowshop Sequencing Problem with Makespan Criterion, 4th Int. Symposium on Intel. Manufac. Syst., Sakarya, Turkey, 442-452. https://doi.org/10. 1007/978-3-540-28646-2 38

[72] Pan, Q.K., Wang, L. \& Qian, B. (2009). A novel differential evolution algorithm for bicriteria no-wait flow shop scheduling problems. Comp. Oper. Res., 36: 2498-2511. https://doi.org/10.1016/j.cor.2008.10.008

[73] Pampara, G., Engelbrecht, A.P. \& Franken, N. (2006). Binary differential evolution. In Proc. IEEE Congress Evol. Comput., 1873-1879.

[74] Lampinen, J. (1999). Differential evolution: New naturally parallel approach for engineering design optimization, in Developments in Computational Mechanics with High Performance Computing, B. H. V. Topping, Ed. Edinburgh, U.K.: Civil-Comp Press, 217-228. https://doi.org/10.4203/ccp.57.11.2

[75] Tasoulis, D.K., Pavlidis, N.G., Plagianakos, V.P. \& Vrahatis, M.N. (2004). Parallel differential evolution. Proc. IEEE Congress Evol. Comput., 2023-2029. https://doi.org/10. $1109 /$ cec.2004.1331145

[76] Yoshida, H. \& Fukuyama, Y. (2018). Parallel multi-population differential evolutionary particle swarm optimization for voltage and reactive power control. IEEJ Trans. Power and Energy, 138 (2): 116-123. https://doi.org/10.23919/sice.2017.8105566

[77] Pedroso, D.M., Bonyadi, M.R. \& Gallagher, M. (2017). Parallel evolutionary algorithm for single and multi-objective optimisation: Differential evolution and constraints handling. Appl. Soft Comput. J., 61: 995-1012. https://doi.org/10.1016/j.asoc.2017.09.006

[78] Madavan, N.K. (2002). Multiobjective optimization using a pareto differential evolution approach. Congress on Evol. Comput., Piscataway, New Jersey, 2: 1145-1150.

[79] Abbass, H.A. \& Sarker, R. (2002). The Pareto differential evolution algorithm. International J. Artif. Intel. Tools, 11(4): 531-552. https://doi.org/10.1142/s0218213002 $\underline{001039}$

[80] Babu, B.V. \& Jehan, M.M.L. (2003). Differential evolution for multiobjective optimization. In Proc. IEEE Congress Evol. Comput., 4: 2696-2703.

[81] Storn, R. (1996). On the usage of differential evolution for function optimization, in Proc. North Am. Fuzzy Inform. Process. Soc., 519-523.

[82] Deb, K. (2000). An efficient constraint handling method for genetic algorithms. Comp. Meth. Appl. Mech. Engine., 186(2-4): 311-338.

[83] Noman, N. \& Iba, H. (2005). Enhancing differential evolution performance with local search for high dimensional function optimization. In Proc. Conf. Genetic Evol. Comput., 967-974. https://doi.org/10.1145/1068009.1068174

[84] Gao, Y. \& Wang, Y. (2007). A memetic differential evolutionary algorithm for high dimensional function spaces optimization. In Proc. 3rd ICNC, 4: 188-192. 
[85] Potter, A.M. \& De Jong, K.A. (1994). A cooperative co-evolutionary approach to function optimization, Proc. of the 3rd Int. Conf. Parallel Problem Solving from Nature, 249-25, Springer-Verlag.

[86] Yang, Z., He, J. \& Yao, X. (2007). Making a difference to differential evolution, in Advances in Metaheuristics for Hard Optimization, Michalewicz, Z. and Siarry, P. Eds., Berlin, Germany: Springer, 415-432.

[87] Yang, Z., Tang, K. \& Yao, X. (2008). Large scale evolutionary optimization using cooperative coevolution, Info. Sci., 178 (15): 2985-2999. https://doi.org/10.1016/j.ins.2008 $\underline{02.017}$

[88] Su, G. (2008). Gaussian process assisted differential evolution algorithm for computationally expensive optimization problems, Pacific-Asia Workshop on Comput. Intel. \& Ind. Appl., 1: 272-276. https://doi.org/10.1109/paciia.2008.184

[89] Zamuda, A., Brest, J., Boskovic, B. \& Zumer, V. (2008). Large Scale Global Optimization using Differential Evolution with self-adaptation and cooperative co-evolution. In Proc. IEEE Congress Evol. Comput., 3718-3725. https://doi.org/10.1109/cec.2008.4631301

[90] Liu, J. \& Lampinen, J. (2005). A fuzzy adaptive differential evolution algorithm. Soft Computing, 9 (6): 448-462. https://doi.org/10.1007/s00500-004-0363-x

[91] Ge, Y.-F., Yu, W.-J., Zhan, Z.-H. \& Zhang, J. (2018). Competition-Based Distributed Differential Evolution. In Proc. IEEE Congress Evol. Comput., Art. no. 8477758.

[92] Das, S., Konar, A. \& Chakraborty, U. (2005). Improved differential evolution algorithms for handling noisy optimization problems. In Proc. IEEE Congress Evol. Comput., 2: 1691-1698. https://doi.org/10.1109/cec.2005.1554892

[93] Mendes, R. \& Mohais, A.S. (2005). DynDE: A differential evolution for dynamic optimization problems. In Proc. IEEE Congress Evol. Comput., 2: 2808-2815. https://doi. org/10.1109/cec.2005.1555047

[94] Brest, J., Zamuda, A., Boskovic, B., Maucec, M.S. \& Zumer, V. (2009). Dynamic optimization using self-adaptive differential evolution. In Proc. IEEE Congress Evol. Comput., 415-422. https://doi.org/10.1109/cec.2009.4982976

[95] Dick, G. \& Whigham, P. (2008). Spatially-structured sharing technique for multimodal problems. J. Comp. Sci. Technol., 23: 64-76. https://doi.org/10.1007/s11390-008-9110-6

[96] Pétrowski, A. (1996). A clearing procedure as a niching method for genetic algorithms. In Proc. IEEE Congress Evol. Comput., New York, USA, 798-803.

[97] Thomsen, R. (2004). Multimodal optimization using crowding-based differential evolution. In Proc. IEEE Congress Evol. Comput., 1382-1389.

[98] Li, J.-P., Balazs, M.E, Parks, G.T. \& Clarkson, P.J. (2002). A species conserving genetic algorithm for multimodal function optimization. Evol. Comput., 10(3): 207-234. https://doi.org/10.1162/106365602760234081

[99] Qu, B.Y. \& Suganthan, P.N. (2010). Novel multimodal problems and differential evolution with ensemble of restricted tournament selection. In Proc. IEEE Congress Evol. Comput., 3480-3486. https://doi.org/10.1109/cec.2010.5586341

[100] Hansen, N. \& Kern, S. (2004). Evaluating the CMA evolution strategy on multimodal test functions. In Proc. 8th Int. Conf. Parallel Problem Solving from Nature, Berlin, Germany, 282-291. https://doi.org/10.1007/978-3-540-30217-9 29

[101] Langdon, W.B. \& Poli, R. (2002). Foundations of Genetic Programming, New York: Springer-Verlag.

[102] Ameca-Alducin, M.-Y., Mezura-Montes, E. and Cruz-Ramírez, N. (2014). Differential evolution with combined variants for dynamic constrained optimization. In Proc. IEEE Congress Evol. Comput., 975-982. https://doi.org/10.1109/cec.2014.6900629 
[103] Cui, L., Huang, Q., Li, G., Yang, S., Ming, Z., Wen, Z., Lu, N. \& Lu, J. (2018). Differential evolution algorithm with tracking mechanism and backtracking mechanism. IEEE Access, 6: 44252-44267. https://doi.org/10.1109/access.2018.2864324

[104] He, Y.-C., Wang, X.-Z., Liu, K.-Q. \& Wang, Y.-Q. (2010). Convergent analysis and algorithmic improvement of differential evolution. J. Software, 21(5): 875-885.

[105] Poikolainen, I. \& Neri, F. (2013). Differential evolution with concurrent fitness based local search. In Proc. IEEE Congress Evol. Comput., 384-391. https://doi.org/10.1109/cec.2013. 6557595

[106] Mallipeddi, R. \& Lee, M. (2015). An evolving surrogate model-based differential evolution algorithm. Appl. Soft Comput. J., 34: 770-787. https://doi.org/10.1016/j.asoc. 2015.06.010

[107] Das, S., Mullick, S.S. \& Suganthan, P.N. (2016). Recent advances in differential evolution - An updated survey. Swarm \& Evol. Comput., 27: 1-30. https://doi.org/10.1016/j.swevo. 2016.01.004

[108] Fan, Q., Yan, X. \& Zhang, Y. (2018). Auto-selection mechanism of differential evolution algorithm variants and its application. Euro. J. Operat. Res., 270 (2): 636-653. https://doi.org/10.1016/j.ejor.2017.10.013

[109] Céspedes-Mota, A., Castañón, G., Martínez-Herrera, A.F. \& Cárdenas-Barrón, L.E. (2016). Optimization of the distribution and localization of wireless sensor networks based on differential evolution approach. Math. Probl. Engine., 2016: Art. no. 7918581. https://doi.org/10.1155/2016/7918581

[110] Kamal, A., Warip, M.N.M., Elshaikh, M. \& Badlishah, R. (2016). Differential evolution (DE) algorithm to optimize Berkeley-MAC protocol for wireless sensor network (WSN). J. Theo. Appl. Info. Tech., 89(2): 314-319.

[111] Zhang, X. \& Zhang, X. (2016). Population-adaptive differential evolution-based power allocation algorithm for cognitive radio networks. Eurasip J. Wireless Comm. Network., 2016(1): Article number 219. https://doi.org/10.1186/s13638-016-0722-1

[112] Lin, C.-C., Tsai, C.-T., Deng, D.-J., Tsai, I.-H. \& Jhong, S.-Y. (2017). Minimizing electromagnetic pollution and power consumption in green heterogeneous small cell network deployment. Computer Networks, 129: 536-547. https://doi.org/10.1016/j.comnet. $\underline{2017.05 .023}$

[113] Liu, Y., Yang, Z., Yu, R., Xiang, Y. \& Xie, S. (2015). An efficient MAC protocol with adaptive energy harvesting for machine-to-machine networks. IEEE Access, 3: 358-367. https://doi.org/10.1109/access.2015.2421517

[114] Rodway, J. \& Musilek, P. (2017). Harvesting-aware energy management for environmental monitoring WSN. Energies, 10(5): Article number 607. https://doi.org/10. $\underline{3390 / \mathrm{en} 10050607}$

[115] Sumithra, S. \& Victoire, T.A. (2015). Differential evolution algorithm with diversified vicinity operator for optimal routing and clustering of energy efficient wireless sensor networks. Scient. World J., 2015: Art. no. 729634. https://doi.org/10.1155/2015/729634

[116] Rajendra Prasad, D., Naganjaneyulu, P.V. \& Satya Prasad, K. (2016). Energy efficient clustering in multi-hop wireless sensor networks using differential evolutionary MOPSO. Brazil. Arch. Biol. Technol., 59: Art. no. e161011. https://doi.org/10.1590/1678-43242016161011

[117] Mittal, N., Singh, U. \& Sohi, B.S. (2017). A Novel Energy Efficient Stable Clustering Approach for Wireless Sensor Networks. Wireless Pers. Comm., 95(3): 2947-2971. https://doi.org/10.1007/s11277-017-3973-1 
[118] Rajendra Prasad, D., Naganjaneyulu, P.V. \& Satya Prasad, K. (2017). A Hybrid Swarm Optimization for Energy Efficient Clustering in Multi-hop Wireless Sensor Network. Wireless Pers. Commun., 94(4): 2459-2471. https://doi.org/10.1007/s11277-016-3562-8

[119] Potthuri, S., Shankar, T. \& Rajesh, A. (2018). Lifetime Improvement in Wireless Sensor Networks using Hybrid Differential Evolution and Simulated Annealing (DESA). Ain Shams Engine. J., 9(4): 655-663. https://doi.org/10.1016/j.asej.2016.03.004

[120] Goudos, S.K., Deruyck, M., Plets, D., Martens, L. \& Joseph, W. (2017). Optimization of Power Consumption in 4G LTE Networks Using a Novel Barebones Self-adaptive Differential Evolution Algorithm. Telecom. Syst. 66(1): 109-120. https://doi.org/10.1007/s $\underline{11235-017-0279-2}$

[121] Liu, N., Plets, D., Goudos, S.K., Martens, L. \& Joseph W. (2015). Multi-objective network planning optimization algorithm: human exposure, power consumption, cost, and capacity. Wireless Networks, 21(3): 841-857. https://doi.org/10.1007/s11276-014-0822-y

[122] Nekooei, S.M., Chen, G. \& Rayudu, R.K. (2017). Automatic design of fuzzy logic controllers for medium access control in wireless body area networks - An evolutionary approach. Appl. Soft Comput. J., 56: 245-261. https://doi.org/10.1016/j.asoc.2017.02.022

[123] Goudos, S.K., Plets, D., Liu, N., Martens, L. \& Joseph, W. (2015). A multi-objective approach to indoor wireless heterogeneous networks planning based on biogeography-based optimization. Computer Networks, 91: 564-576. https://doi.org/10.1016/j. comnet.2015.08.037

[124] Wang, L. An, L., Ni, H.Q., Ye, W., Pardalos, P.M. \& Fei, M.R. (2016). Pareto-based multi-objective node placement of industrial wireless sensor networks using binary differential evolution harmony search. Adv. Manufacturing, 4(1): 66-78. https://doi.org/10.1007/s40436-016-0135-8

[125] Cao, B., Zhao, J., Yang, P., Lv, Z., Liu, X., Kang, X., Yang, S., Kang, K. \& AnvariMoghaddam, A. (2018). Distributed parallel cooperative coevolutionary multi-objective large-scale immune algorithm for deployment of wireless sensor networks. Future Gen. Comput. Syst., 82: 256-267. https://doi.org/10.1016/j.future.2017.10.015

[126] Ayinde, B.O. \& Hashim, H.A. (2018). Energy-Efficient Deployment of Relay Nodes in Wireless Sensor Networks Using Evolutionary Techniques. Int. J. Wireless Info. Networks, 25(2): 157-172. https://doi.org/10.1007/s10776-018-0388-1

[127] Huang, L., Liu, J. \& Guo, L. (2018). A hybrid mutation artificial bee colony algorithm for spectrum sharing. Int. J. High Perf. Comput. Network., 12(3): 299-306. https://doi.org/10.1504/ijhpcn.2018.10016377

[128] Khedher, M., Liouane, H. \& Douik, A. (2018). Optimized minimum spanning tree for border nodes selection in wireless sensor networks. 15th International Multi-Conference on Systems, Signals and Devices, Art. no. 8570484, 660-665. https://doi.org/10.1109/ssd. $\underline{2018.8570484}$

[129] Merlin Sheeba, G. \& Nachiappan, A. (2016). Fuzzy differential evolution based gateway placements in WMN for cost optimization. Adv. Intel. Syst. Comput., 385: 137-145. https://doi.org/10.1007/978-3-319-23258-4_13

[130] Swayamsiddha, S., Parija, S., Sahu, P.K. \& Singh, S.S. (2017). Optimal reporting cell planning with binary differential evolution algorithm for location management problem. Int. J. Intel. Syst. Appl., 9(4): 23-31. https://doi.org/10.5815/ijisa.2017.04.03

[131] Merlin Sheeba, G. \& Nachiappan, A. (2017). Performance evaluation of fuzzy DE based node placement in WMN. J. Engine. Research, 5(4): 106-120.

[132] Goudarzi, S., Hassan, W.H., Anisi, M.H. \& Soleymani, S.A. (2016). Comparison between hybridized algorithm of GA-SA and ABC, GA, DE and PSO for vertical-handover in het- 
erogeneous wireless networks. Sadhana - Acad. Proc. Engine. Sci., 41(7): 727-753. https://doi.org/10.1016/j.neucom.2016.08.136

[133] Chiu, C.-C., Chen, C.-H., Cheng, Y.-T., Lee, Y.-L., \& Chou, Y.-K. (2018). Beamforming techniques at both transmitter and receiver for indoor wireless communication. J. Appl. Sci. Engine., 21(3): 407-412.

[134] Yu, G., Ma, H. \& Witarsyah, D. (2018). Optimal path selection algorithm for mobile beacons in sensor network under non-dense distribution. Open Physics, 16(1): 1066-1075. https://doi.org/10.1515/phys-2018-0127

[135] Chiu, C.-C., Lai, G.-D. \& Cheng, Y.-T. (2018). Self-adaptive dynamic differential evolution applied to BER reduction with beamforming techniques for ultra wideband MUMIMO systems. Prog. Electromag. Res. 87: 187-197. https://doi.org/10.2528/pierc 18082302

[136] Chiu, C.C., Tong, Y.X. \& Cheng, Y.T. (2019). Comparison of self-adaptive dynamic differential evolution and particle swarm optimization for smart antennas in wireless communication. Int. J. Commun. Syst., Art. no. e3941. https://doi.org/10.1002/dac.3941

[137] Afzal, Z., Shah, P.A., Awan, K.M. \& Zahoor-ur-Rehmanb. (2019). Optimum bandwidth allocation in wireless networks using differential evolution. J. Ambient Intel. Hum. Comput., 10(4): 1401-1412. https://doi.org/10.1007/s12652-018-0858-4

[138] Goudarzi, S., Hassan, W.H., Anisi, M.H. \& Soleymani, S.A. (2017). MDP-Based Network Selection Scheme by Genetic Algorithm and Simulated Annealing for Vertical-Handover in Heterogeneous Wireless Networks. Wireless Pers. Commun., 92(2): 399-436.

[139] Zhao, K. \& Sun, Y. (2017). Genetic-optimization framework for SVC transmission based on partial cooperative communication. J. Syst. Engine. Electro., 28(5): Art. no. 8240030, 861-870. https://doi.org/10.1007/s11277-016-3549-5

[140] Sapre, S. \& Mini, S. (2018). Moth Flame Based Optimized Placement of Relay Nodes for Fault Tolerant Wireless Sensor Networks, In Proc. 9th ICCCNT, Art. no. 8494123. https://doi.org/10.1109/iccent.2018.8494123

[141] Chakraborty, D. (2015). I-QCA: An intelligent framework for quality of service multicast routing in multichannel multiradio wireless mesh networks. Ad Hoc Networks, 33: 221232. https://doi.org/10.1016/j.adhoc.2015.05.007

[142] Chakraborty, D. \& Debbarma, K. (2017). Q-CAR: an intelligent solution for joint QoS multicast routing and channel assignment in multichannel multiradio wireless mesh networks. Appl. Intel., 47(1): 13-27. https://doi.org/10.1007/s10489-016-0871-2

[143] You, K., Yang, W., Yuan, X. \& Wang, Y. (2018). Differentiated services routing protocol of WMSNs for underground coal mines. J. Huazhong Uni. Sci. Technol., 46(9), 1-8.

[144] Murugeswari, R. \& Radhakrishnan, S. (2016). Discrete multi-objective differential evolution algorithm for routing in wireless mesh network. Soft Computing, 20(9): 3687-3698. https://doi.org/10.1007/s00500-015-1730-5

[145] Anumandla, K.K., Peesapati, R. \& Sabat, S.L. (2015). Field programmable gate array implementation of spectrum allocation technique for cognitive radio networks. Comp. Elect. Engine., 42: 178-192. https://doi.org/10.1016/j.compeleceng.2014.07.013

[146] Sharma, N. \& Anpalagan, A. (2015). Differential evolution aided adaptive resource allocation in OFDMA systems with proportional rate constraints. Appl. Soft Comput. J., 34: 3950. https://doi.org/10.1016/j.asoc.2015.04.056

[147] Sachan, R., Choi, T.J. \& Ahn, C.W. (2016). A Genetic Algorithm with Location Intelligence Method for Energy Optimization in 5G Wireless Networks. Disc. Dyna. Nature Soc., 2016: Art. no. 5348203.

[148] Wu, H. (2016). Intelligent traffic scheduling algorithm based on hybrid differential evolution strategy. Int. J. Simul. Syst. Sci. Tech., 17(40): 20.1-20.5. 
[149] El Moiz Dahi, Z.A., Mezioud, C. \& Draa, A. (2017). A 0-1 bat algorithm for cellular network optimisation: A systematic study on mapping techniques. Int. J. Reasoning-based Intel. Syst., 9(1): 22-42. https://doi.org/10.1504/ijris.2017.10007147

[150] Baumgartner, P., Bauernfeind, T., Biro, O., Magele, C., Renhart, W. \& Torchio, R. (2018). Synthesis of NFC antenna structure under multi-card condition. International Applied Computational Electromagnetics Society Symposium in Denver, ACES-Denver. https://doi.org/10.23919/ropaces.2018.8364095

[151] Baumgartner, P., Bauernfeind, T., Biro, O., Magele, C., Renhart, W. \& Torchio, R. (2018). Synthesis of NFC antenna structure under multi-card condition. Appl. Comput. Electro. Society J., 33(10): 1161-1163. https://doi.org/10.23919/ropaces.2018.8364095

[152] Sohail, M.F., Ghauri, S.A. \& Alam, S. (2017). Channel Estimation in Massive MIMO Systems Using Heuristic Approach. Wireless Pers. Commun., 97(4): 6483-6498. https://doi.org/10.1007/s11277-017-4849-0

[153] Zheng, S., Gao, S., Yin, Y., Luo, Q., Yang, X., Hu, W., Ren, X. \& Qin, F. (2018). A Broadband Dual Circularly Polarized Conical Four-Arm Sinuous Antenna. IEEE Trans. Antennas Prop., 66(1): Art. no. 8103784, 71-80. https://doi.org/10.1109/tap.2017.2772301

[154] Harikrishnan, R., Jawahar Senthil Kumar, V. \& Sridevi Ponmalar, P. (2016). A Comparative Analysis of Intelligent Algorithms for Localization in Wireless Sensor Networks. Wireless Pers. Commun., 87(3): 1057-1069. https://doi.org/10.1007/s11277-015-2635-4

[155] Liu, D., Zhang, L., Bing, X., Shao, Y. \& Xu, B. (2017). Localization Method Based on Modified Cuckoo Difference Optimization for Wireless Sensor Networks. J. Syst. Simul., 29(4): 791-797.

[156] Sivakumar, S. \& Venkatesan, R. (2016). Performance evaluation of hybrid evolutionary algorithms in minimizing localization error for wireless sensor networks. J. Scient. Indust. Res., 75(5): 289-295.

[157] Zhou, C., Chen, L., Chen, Z., Li, X. \& Dai, G. (2017). A sine cosine mutation based differential evolution algorithm for solving node location problem. Int. J. Wireless Mobile Comput., 13(3): 253-259. https://doi.org/10.1504/ijwmc.2017.10009499

[158] Sachan, R., Muhammad, Z., Jeong, J., Ahn, C.W. \& Youn, H.Y. (2017). MABC: PowerBased Location Planning with a Modified ABC Algorithm for 5G Networks. Disc. Dyn. Nature Soc., 2017: Art. no. 4353612. https://doi.org/10.1155/2017/4353612

[159] SrideviPonmalar, P., Jawahar Senthil Kumar, V. \& Harikrishnan, R. (2017). Hybrid firefly variants algorithm for localization optimization in WSN. Int. J. Comput. Intel. Syst., 10(1): 1263-1271. https://doi.org/10.2991/ijcis.10.1.85

[160] Cui, L., Xu, C., Li, G., Ming, Z., Feng, Y. \& Lu, N. (2018). A high accurate localization algorithm with DV-Hop and differential evolution for wireless sensor network. Appl. Soft Comput. J., 68: 39-52. https://doi.org/10.1016/j.asoc.2018.03.036

[161] Qin, M. \& Zhu, R. (2018). A Monte Carlo localization method based on differential evolution optimization applied into economic forecasting in mobile wireless sensor networks. Eurasip J. Wireless Commun. Network., 2018(1): Art. no. 32. https://doi.org/10.1186/s1 3638-018-1037-1

[162] Srideviponmalar, P., Jawahar Senthil Kumar, V. \& Harikrishnan, R. (2018). Hybrid genetic algorithm-differential evolution approach for localization in WSN. Adv. Intel. Syst. Comput., 695: 263-271. https://doi.org/10.1007/978-981-10-7566-7 27

[163] Vedesh, P., Shivani, T. \& Bagadi, K.P. (2018). Efficient Implementation of localization in Wireless Sensor Networks Using Optimization Techniques. Proc. 2nd ICECA, Art. no. 8474913, 1332-1337. https://doi.org/10.1109/iceca.2018.8474913 
[164] Yu, G. (2017). A dual population based firefly algorithm and its application on wireless sensor network coverage optimization. Int. J. Wirel. Mobile Comput., 13(3): 188-192. https://doi.org/10.1504/ijwmc.2017.10009494

[165] Qiao, D. \& Pang, G.K.H. (2016). A Modified Differential Evolution with Heuristic Algorithm for Nonconvex Optimization on Sensor Network Localization. IEEE Trans. Vehic. Technol., 65(3): Art. no. 7055895, 1676-1689. https://doi.org/10.1109/tvt.2015.2409319

[166] Cevik, T. \& Gunagwera, A. (2018). Coverage and energy efficiency optimization for randomly deployed multi-tier wireless multimedia Sensor Networks. Int. J. Comm. Netw. Info. Security, 10(1): 28-36.

[167] Wang, Y.J. \& Li, X.J. (2016). Targets coverage algorithm based on three-dimensional directional sensing model. J. Info. Hiding Multimed. Signal Process., 7(1): 145-152.

[168] Zhang, Q., \& Fok, M.P. (2017). A two-phase coverage-enhancing algorithm for hybrid wireless sensor networks. Sensors, 17(1): Art. no. 117. https://doi.org/10.3390/s17010117

[169] Qin, N.N. \& Chen, J.L. (2018). An area coverage algorithm for wireless sensor networks based on differential evolution. Int. J. Dist. Sensor Networks, 14(8): 1-11. https://doi.org/10.1177/1550147718796734

[170] Xu, Y., Ye, Y., Zhang, H., Zhang, W. \& Lv, Y. (2019). A fast two-objective differential evolution for the two-objective coverage problem of WSNs. Memetic Computing, 11(1): 89-107. https://doi.org/10.1007/s12293-018-0264-7

[171] Céspedes-Mota, A., Castañón, G., Martínez-Herrera, A.F., Cárdenas-Barrón, L.E. \& Sarmiento, A.M. (2018). Differential evolution algorithm applied to wireless sensor distribution on different geometric shapes with area and energy optimization. J. Net. Comp. Appl., 119: 14-23. https://doi.org/10.1016/j.jnca.2018.06.006

[172] Yang, J., Xu, Y., Wei, C. \& Jiang, S. (2016). A gateway deployment algorithm in cyberphysical system based on differential evolution. J. Electro. Info. Tech., 38(1): 195-201.

[173] Cao, B., Kang, X., Zhao, J., Yang, P., Lv, Z. \& Liu, X. (2018). Differential EvolutionBased 3-D Directional Wireless Sensor Network Deployment Optimization. IEEE Internet of Things J., 5(5): Art. no. 8279426, 3594-3605. https://doi.org/10.1109/jiot.2018.2801623

[174] Wu, D. \& Du, F. (2018). A Parallel Ranging-Based Relative Position and Orientation Measurement Method for Large-Volume Components. J. Sensors, 2018: Art. no. 2404825. https://doi.org/10.1155/2018/2404825

[175] Ren, G., Wu, J. \& Versonnen, F. (2018). Bee-based reliable data collection for mobile wireless sensor network. Cluster Computing, 10.1007/s10586-018-2116-0. https://doi.org/ $\underline{10.1007 / \mathrm{s} 10586-018-2116-0}$

[176] Rajalakshmi, S. \& Maguteeswaran, R. (2015). Quality of Service Routing in Manet Using a Hybrid Intelligent Algorithm Inspired by Cuckoo Search. Scient. World J., 2015: Art. no. 703480. https://doi.org/10.1155/2015/703480

[177] Wan, Q., Weng, M.J. \& Liu, S. (2019). Optimization of wireless sensor networks based on differential evolution algorithm. Int. J. Online Engine., 15(1): 183-195. https://doi.org/10 .3991/ijoe.v15i01.9786

[178] Subramanian, M. \& Jaisankar, N. (2018). An optimal path selection in a clustered wireless sensor network environment with swarm intelligence-based data aggregation for air pollution monitoring system. Int. J. Comp. Aided Engine. Tech., 10(4): 378-403. https://doi.org/10.1504/ijcaet.2018.10012349

[179] Xue, Y., Jia, W., Zhao, X. \& Pang, W. (2018). An Evolutionary Computation Based Feature Selection Method for Intrusion Detection. Security Comm. Networks, 2018: Art. no. 2492956. https://doi.org/10.1155/2018/2492956 
[180] Ranjith Kumar, A. \& Sivagami, A. (2018). Security Aware Multipath Routing Protocol for WMSNs for Minimizing Effect of Compromising Attacks. J. Netw. Syst. Magt. https://doi. org/10.1007/s10922-018-9477-9

[181] Basha, A.R. \& Yaashuwanth, C. (2018). Double secure optimal partial aggregation using trust inference and hybrid syncryption algorithm for wireless sensor networks. J. Comput. Theo. Nanosci., 15(2): 423-436. https://doi.org/10.1166/jctn.2018.7106

[182] Guo, W., Chen, Y., Cai, Y., Wang, T. \&Tian, H. (2017). Intrusion detection in WSN with an improved NSA based on the DE-CMOP. KSII Transac. Internet and Info. Syst., 11(11): 5574-5591. https://doi.org/10.3837/tiis.2017.11.022

[183] Xu, Z., Gu, R., Huang, T., Xiang, H., Zhang, X., Qi, L. \& Xu, X. (2018). An IoT-oriented offloading method with privacy preservation for cloudlet-enabled wireless metropolitan area networks. Sensors, 18(9): Art. no. 3030. https://doi.org/10.3390/s18093030

[184] Chen, W., Zhao, H., Li, T. \& Liu, Y. (2018). Optimal probabilistic encryption for distributed detection in wireless sensor networks based on immune differential evolution algorithm. Wireless Networks, 24(7): 2497-2507. https://doi.org/10.1007/s11276-017-1484-3

[185] Liu, H. (2019). SINR-based multi-channel power schedule under DoS attacks: A Stackelberg game approach with incomplete information. Automatica, 100: 274-280. https://doi.org/10.1016/j.automatica.2018.11.034

[186] Wisittipanich, W. \& Buakum, D. (2019). Optimal design of pipe diameter in water distribution system by multi-objective differential evolution algorithm: A case study of small town in Chiang Mai. Lect. Notes Elect. Engine., 513: 351-360. https://doi.org/10. 1007/978-981-13-1059-1_33

[187] Fu, H., Feng, S., Liu, J. \& Tang B. (2016). The modeling and simulation of gas concentration prediction based on De-Eda-Svm. Chinese J. Sensors Actua., 29(2): 285-289.

[188] Prauzek, M., Krömer, P., Rodway, J. \& Musilek, P. (2016). Differential evolution of fuzzy controller for environmentally-powered wireless sensors. Appl. Soft Comput. J., 48: 193206. https://doi.org/10.1016/j.asoc.2016.06.040

[189] Mahmoudzadeh, S., Powers, D.M.W. \& Atyabi, A. (2018). UUV's Hierarchical DE-Based Motion Planning in a Semi Dynamic Underwater Wireless Sensor Network. IEEE Trans. Cyber., DOI: 10.1109/TCYB.2018.2837134. https://doi.org/10.1109/tcyb.2018.2837134

[190] Céspedes-Mota, A., Castañón, G., Martínez-Herrera, A.F. \& Cárdenas-Barrón, L.E. (2018). Multiobjective Optimization for a Wireless Ad Hoc Sensor Distribution on Shaped-Bounded Areas. Math. Probl. Engine., 2018: Article no. 7873984. https://doi.org/10.1155/2018/7873984

[191] Hao, X., Wang, L., Liu, J., Xie, L. \& Zhang, W. (2018). Resource allocation optimization algorithm based on double populations differential evolution in WSN. J. Communications, 39(4): 68-75.

\section{Authors}

Hilary I. Okagbue received the B.Tech degree in Industrial and Applied Mathematics from the Federal University of Technology, Owerri, Nigeria and M.Sc degree in Statistics from the University of Lagos, Akoka, Nigeria in 2007 and 2010 respectively. He is a doctoral student of the department of Mathematics, Covenant University, Ota, Nigeria. His research interests include: Quantile approximation models, nonlinear statistical modeling and data analysis. He has some papers in some learned journals. 
Muminu O. Adamu received the B.Sc (Hons) degree in Mathematics/Statistics, M.Sc degree in Statistics and Ph.D degree in Statistics from the University of Lagos, Akoka, Nigeria in 1995, 1998 and 2005 respectively. Since 2004, he has been a fulltime lecturer and researcher in the department of Mathematics, University of Lagos, Akoka, Nigeria and now has risen to the rank of an associate professor of statistics. His research interests include: combinatorial optimization, operations research with emphasis on machine scheduling and applied statistics. He is a reviewer for the following journals: Journal of Applied Mathematics, IEEE Transactions on Evolutionary Computation and Journal of Nigerian Mathematical Society.

Timothy A. Anake received the B.Sc degree in Mathematics from the University of Uyo, Uyo, Nigeria, M.Sc degree in Mathematics from the University of Ibadan, Ibadan, Nigeria and Ph.D degree in Mathematics in Mathematics from Covenant University, Ota, Nigeria in 2000, 2004 and 2011 respectively. He was a lecturer at Igbinedion University, Okada, Nigeria from 2004 to 2006. Since 2006, he has been a full-time lecturer and researcher in the department of Mathematics, Covenant University, Ota, Nigeria and now has risen to the rank of an associate professor of numerical analysis and computation. His research interests include: numerical solutions of differential equations, nonlinear modeling of engineering systems and numerical approximation of physical systems.

Article submitted 2019-04-12. Resubmitted 2019-05-09. Final acceptance 2019-05-11. Final version published as submitted by the authors. 